\title{
CONTROVERSIA EN TORNO A LA FIGURA DE KATE EN THE TAMING OF THE SHREW DE WILLIAM SHAKESPEARE
}

\author{
Alicia ROMERO LÓPEZ \\ Westfälische Wilhelms-Universitat Münster \\ aliciafilol@gmail.com
}

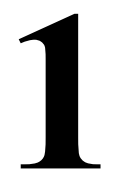

\section{Introducción}

Las interpretaciones del personaje de Katherina en la obra The Taming of the Shrew de William Shakespeare son muchas y muy variadas, convirtiéndola así en una de las figuras femeninas más controvertidas de la obra shakesperiana. Tras una primera lectura de la obra uno de los elementos más destacables, desde la perspectiva actual, es la misoginia, remarcada sobre todo en el discurso final de Kate. Con este trabajo queremos mostrar que el lector debe ir más allá de esa primera impresión y revisar tanto el tipo de texto al que nos enfrentamos como el contexto y la época en la que esta obra se represente. Para entender cómo fue la recepción de la misma a finales del XVI, ya que fue estrenada entre 1591 y 1592, aunque no se imprimió hasta 1623 (Shakespeare 2008: VIII), consideramos de gran importancia el analizar brevemente el papel de la mujer en la época. La situación de las mujeres en el Renacimiento, no solo inglés, era bastante desfavorable, siendo las cualidades básicas de la misma la sumisión y el silencio. Los manuales de conducta de la «buena mujer»o «mujer natural» (aquella que es madre y esposa) estaban a la orden del día en la época, un claro ejemplo es $L a$ formación de la mujer casada de Luis Vives.

Una de las claves para entender The Taming of the Shrew es la relevancia que se da al poder del matrimonio y la fuerza que este tenía en la época. Vives estructura su obra La formación de la mujer cristiana según tres arquetipos femeninos: doncella, casada o viuda (1994a). El pensamiento humanista difundía la idea del matrimonio como una unidad social, pero no la establece en términos de igualdad. Las jerarquías domésticas siguen existiendo y las obligaciones de cada uno de los cónyuges están claramente diferenciadas: «God hath placed her neere, \& so indeed she ist next to the husband; but yet still he is the head, and her obedience must be his crowne, the grace, the creditoe, the ornament of his goverment...» (Hieron, 1620: 407)1 . En 1947 la Iglesia Anglicana produjo una serie de textos, tales como sermones y homilías con los que difundir sus ideas en Inglaterra y en el Segundo

\footnotetext{
${ }^{1}$ Samuel Hieron (1572-1592), predicador puritano y escritor nacido en Inglaterra. Estudió en el colegio Eton. Sus obras son generalmente sermones y comentarios (Chalmers, 1814: 461).
} 
Tomo de Homilías (1563) aparece ya el tema del matrimonio (A Homily of the State of Matrimony), el cual se va a mostrar según lo anteriormente expuesto: el matrimonio es entendido como una unión amistosa, pero previendo que ésta va a estar cargada de luchas de poder (Kemp, 2010: 195-196). Lo que llama la atención de este texto, desde la perspectiva actual, es que en él está presente lo que hoy en día podríamos denominar «violencia de género», la cual debía ser tan frecuente en la época que hasta los propios tratadistas morales la criticaban: «the sermon also discourages husbands from beating their wives» (Kemp, 2010: 196). El capítulo IX de la obra Los deberes del marido de Luis Vives es titulado: «La represión y la corrección»; sin embargo, en él tampoco se incita a la violencia contra la mujer, sino que va a intentar acercarse a la idea del matrimonio como unión ideal: «Reprenderás a tu esposa de tal modo que ella comprenda que la reconvención nace de la solicitud de tu amor, porque deseas que sea mejor y quieres que desaparezca de ella toda clase de vicio para que la llama del amor conyugal arda con más calor y pureza» (Vives, 1994b). Al ser la mujer de una inferior naturaleza se aconseja soportar sus actitudes altivas y se insta a enseñarle cuál es el comportamiento adecuado sin hacer uso de la violencia: «Si una esposa repetidamente advertida, reprendida y censurada, se muestra inasequible a la persuasión, y demasiado dura para poder reconducir su conducta, mientras guarde íntegra su castidad, sopórtesela: no hay que romper el arco por tensarlo demasiado» (Vives 1994b). Vives apoya sus argumentos en la Biblia, citando la Epístola a los Efesios en la que se dice que marido y mujer son una misma carne (Eph, 5, 31).

En cuanto al comportamiento de la mujer para con su marido Vives vuelve a citar la Biblia, como refuerzo a sus ideas, y en el capítulo IV del libro II, «Cómo deberá comportarse la esposa con el marido», de La formación de la mujer cristiana pone como ejemplo la Epístola a Timoteo, cuyo capítulo II se titula: «Compostura de las mujeres»; en él se presentan las bases de la obediencia de la mujer al marido: «La mujer oiga la instrucción en silencio con total sumisión. No permito que la mujer enseñe ni que domine al hombre. Que se mantenga en silencio. Porque Adán fue formado primero y Eva en segundo lugar» (Tim. 2, 11-13). Vives no es interesante solo por el papel como moralista que desempeñó en la época que nos atañe, sino por el alcance que tuvo, pues incluso Shakespeare llegó a conocer sus textos:

Shakespeare siguió, en la escuela de gramática de Stratford-on-Avon, el sistema de San Pablo, aunque sin duda como concesión a la influencia de Eton, usó el Satellitium de Vives por la indudable influencia que se refleja en obras como Merchant of Venice, Corialanus, Twelfth Nigth, The Tempest, Love's Labour's Lost y Hamlet. Hay clara evidencia del uso de la Introductio ad sapientiam en, al menos, cinco escuelas de la época Tudor que siguieron de modo coincidente el sistema de Eton (Roca y Gómez-Hortigüela, eds. 2001: IV).

\section{La figura de Katherina en The Taming of the Shrew}

Todo lo explicitado anteriormente se muestra de forma clara en la obra The Taming of the Shrew, en la que se presentan aspectos tales como el silencio en la mujer o el no usar la violencia física como forma de represión. Katherina, en un primer momento, está fuera del marco social al que la mujer debía estar subordinada, pues una de sus características es el ser dueña de la palabra, con la cual juega, grita 
e insulta: «her name is Katherina Minola, / renowned in Padua for her scolding tongue» $(181)^{2}$. Es esta independencia, esta fuerza y este intento de ruptura con las normas sociales lo que va a hacer que sea considerada como una bravía, una furia (traducciones mucho más fieles al original que la que se ha usado normalmente: fierecilla): «What, shall I be appointed hours, as thought, belike, / I knew not what to take, and what to leave? Ha!» (166). La «doma» de Kate va a estar apoyada por tres bandos, por un lado por los enamorados de Blanca, que no podrán intentar casarse con ella hasta que su hermana lo haya hecho; por el padre de las dos hermanas, que considera que su hija mayor es demasiado brava y quiere traspasar los poderes al marido (recordemos que en esta época la mujer pasaba de ser propiedad del padre a ser propiedad del marido) y por otro lado por Petruccio, el «domador», al cual le mueve solamente el beneficio económico que puede sacar del matrimonio con Kate. Por lo que nos encontramos con una joven que intentando escapar de la norma social se ve rodeada a tres bandas que la quieren domesticar y hacer que se adapte a los convencionalismos de la época. Petruccio, decidido a tener a Kate, consigue el consentimiento del padre de la joven y la obligan a casarse con él: «Thus in plain terms: your father hath consented / That you shall be my wife; your dowry 'greed on, / And will you, nill you, I will marry you» (210). Consideramos que el comienzo de la «doma» es anterior a la boda, pues Petruccio intenta enamorar a Kate respondiendo con palabras dulces y agradables a sus malas contestaciones, si bien esta actitud cambia desde el momento que llega tarde a su propia boda y vestido de forma harapienta, está decidido a someter a Kate: «For I am he am born to tame you, Kate, / And bring you from a wild Kate to a Kate / Conformable as other household Kates» (211).

Un momento determinante, usado posteriormente para la interpretación de la obra, se sucede cuando Petruccio tras casarse con Kate decide prescindir del banquete nupcial y marcharse a sus tierras. Kate en este momento parece sucumbir al amor y le ruega que se quede con ella: «Now if you love me, stay» (237). Al no ceder ante sus ruegos Kate vuelve a su estado habitual: «Do what thou canst, I will not go today, / No, nor tomorrow - not till I please myself. / The door is open, sir, there lies your way» (237), pero finalmente parte junto su nuevo marido. A partir de este momento comienzan los actos de violencia, y aunque no le pone la mano encima a Kate (en conjunción con lo dispuesto por los tratadistas morales de la época), sí podemos hablar de violencia psicológica y de cierta forma de violencia física. Las palizas que da Petruccio a sus criados funcionan como una amenaza contra Kate, pues si ella no hace lo que su marido quiere podrá ser víctima de las mismas ${ }^{3}$. Si unimos esta intimidación psicológica a la inanición y falta de sueño a la que es sometida la protagonista observamos que la doma de la joven, careciendo de golpes, es brutal ${ }^{4}$ :

\footnotetext{
${ }^{2}$ Las citas referidas a la obra The Taming of the Shrew de W. Shakespeare serán tomadas de la edición de Barbara Hodgdon (Shakesperare, 2010). Se citarán tan solo con el número de página.

${ }^{3}$ Esta forma de intimidación recuerda mucho a la usada el Cuento XXXV, «De lo que contesçió a un mançebo que casó con una mujer muy fuerte y muy bravía», de El Conde Lucanor.

${ }^{4}$ En el poema, A Merry Jest of a Shrewd and Curst Wife..., usado como fuente por Shakespeare, la violencia física sí es explícita.
} 


\title{
Alicia Romero López
}

\author{
Another way I have to man my haggard, \\ To make her come and know her keeper's call. \\ That is, to watch her, as we watch these kites \\ That bate, and beat, and will not be obedient. \\ She ate no meat today, nor none shall eat. \\ Last night she slept not, nor tonight she shall not (251-252).
}

Es por todo ello por lo que en una primera lectura del texto el lector no se sorprende con el monólogo final de Kate en el que los tres amantes deciden apostar cuál de las mujeres es la más sumisa, Kate, Blanca o la viuda. Tras la respuesta negativa de las dos últimas, es Kate la que va a acudir a «la llamada del amo» e incluso va a insultar a las otras: «Come, come, you froward and unable worms» (303). Un discurso en el que la sumisión de la mujer queda plasmado en boca de una «rebelde» hace que esta subyugación marital sea, aún si cabe, más impactante: «Thy husband is thy lord, thy life, thy keeper, / Thy head, thy sovereign, one that cares for thee» (302). El texto shakesperiano acaba con la alabanza de Lucentio a Petruccio, por haber sabido «domar» a su mujer, por lo tanto la obra parece tener como moraleja que el saber dominar a la mujer da prestigio: «Tis a wonder, by your leave, she will be tamed so» (305). Tras este duro final, la misoginia textual parece clara, sin embargo, debemos tener en cuenta diferentes aspectos que pueden hacer que entendamos la obra en su conjunto desde otro punto de vista.

\section{1. Katherina: ¿sumisión o subversión?}

La interpretación del texto shakesperiano se mueve entre extremos opuestos, por ser una obra de marcada controversia, y esta dependerá de la lectura que de ella se haga o del montaje teatral al que asistamos. Incluso en la época de Shakespeare fue esta una obra que produjo ideas encontradas, es por ello por lo que aparecieron «respuestas dramáticas» como la obra de John Fletcher, The Tamer Tamed o A Woman's Prize (escrita entre 1609-10), en la que Katherina es asesinada durante la doma a manos de Petruccio (Kemp, 2010: 76). Este giro de la historia, al quitar la victoria de Petruccio sobre Kate, hace pensar que Fletcher entendió el monólogo final como un discurso de sumisión total de la mujer (Shakespeare, 2008: XIV). Aunque para la crítica actual esta no ha sido una idea de consenso y el monólogo final se ha entendido desde una perspectiva discursiva misógina hasta una feminista. Por un lado se podría pensar que el comportamiento «furioso» de Kate vendría dado por las condiciones sociales que la rodean: su hermana es la que ha ganado el favor del padre y el de la sociedad, siendo esa mujer ideal, bella, silenciosa, sumisa... y Kate, que se rebela ante esta actitud, subvierte la norma establecida. Un apunte magnífico que hace Callaghan en su Introducción a esta obra de Shakespeare es que siendo Blanca la mujer perfecta que se atiene a los ideales de la época, sin embargo, va a ser Kate la que se case con Petruccio obligada por su padre, mientras que Blanca termina casándose con quien ella realmente ama (Shakespeare, 2008: XIII), apareciendo así también como un personaje subversivo dentro de la obra.

En cuanto a las interpretaciones y críticas más recientes del texto shakesperiano nos encontramos con una de George Bernard Shaw, quien escribió una carta a The Pall Mall Gazette, el 8 de junio de 
1888, bajo un pseudónimo femenino, en la que criticaba duramente la obra: «a piece which is one vile insult to womanhood and manhood from the first word to the least» (cit. en Shakespeare, 2010: 140). Contraria a esta opinión es la de Harold Bloom, que alega que Kate está perdidamente enamorada de Petruccio y apoya la teoría de que finalmente la que gana es ella: «Petruchio gets to swagger, and Kate will rule him and the household, perpetually acting her role as the reformed shrew» (Bloom, 1998). La teoría de Bloom es demasiado amable y a la hora de tratar las vejaciones a las que Kate es sometida, lo hace de pasada y sin darle importancia, para reforzar así la idea de que es ella la que se impone al hombre. Si bien consideramos que en esta obra nos enfrentamos a mucho más que a un simple monólogo misógino, rebajar las penurias de Kate no es lícito, máxime teniendo en cuenta todo lo anteriormente referido respecto a la sociedad de la época y la normalidad de la violencia contra la mujer.

Siguiendo esta línea de pensamiento consideramos que hay dos factores determinantes para poder analizar de forma más profunda el monólogo final: por un lado tendríamos el marco externo con el que comienza la obra, es decir, la historia del borracho Sly (metateatro) y por otro lado la ironía que se desprende del discurso de Kate. El uso de la Induction, muchas veces suprimida por los directores, juega un papel determinante, ya que es un recurso con el que Shakespeare juega para añadir ese contraste entre realidad e irrealidad, afianzando los elementos cómicos. Al enmarcar la obra The Taming of the Shrew dentro de una escena en la que se engaña a un pobre borracho para que crea que es un gran señor, se juega desde el comienzo del texto con la contraposición entre realidad y sueño, lo que va a distanciar al espectador de la obra que va a ser representada para el pobre Sly. Pero no solo se da este distanciamiento con el «teatro dentro del teatro», sino que este marco externo va a quedar abierto, pues la obra termina sin retomar la historia de Sly. Esta apertura del final incrementa la duda en el lector/espectador sobre lo que es verdad y lo que es mentira. Si tenemos en cuenta esto a la hora de leer el monólogo final, la primera idea misógina que nos habremos hecho del mismo varía, pues ahora no sabemos a qué nos estamos enfrentando. Además hay que tener en cuenta la ironía que irradia el monólogo de Kate (sin olvidar que estamos ante una comedia), un ejemplo que refuerza esta idea es el momento en el que Kate afirma: «And for thy maintenance commits his body / To painful labour both by sea and land, / To watch the night in storms, the day in cold, / While thou liest warm at home, secure and safe» (302), pues sabemos que Petruccio es un noble y nunca va a estar en tales situaciones. Para Booth la ironía en este texto sería clara: «Whenever a story, play, poem, or essay reveals what we accept as a fact and then contradicts it, we have only two posibilities. Either the author has been careless or he has presented us with an inescapable ironic invitation» (1974: 61).

Teniendo en cuenta los dos aspectos anteriormente mencionados podemos leer el monólogo final en clave no misógina. Nuestra opinión se mueve entre la de Shaw y la de Bloom: la del primero se podría decir que es exagerada, pues no ha tenido en cuenta estos dos aspectos y quizá se ha quedado en una lectura de base misógina (entendible también por la época en la que está escrita) y la de Bloom deja totalmente de lado la misoginia, algo que consideramos improbable por ser una obra de finales del XVI. Se podría decir que esta obra de Shakespeare lo que hace es enfrentar al espectador con la 
realidad social de la época. Está claro que en los siglos XVI y XVII el espectador se encontraría con la representación de ciertas escenas perfectamente asumibles como reales, pero desgraciadamente tanto aquellos espectadores como los de hoy en día nos hemos encontrado con la misma realidad: la violencia de género. Por lo tanto esta obra nos enfrenta con el día a día y nos pone frente a frente con una sociedad patriarcal que trata a las mujeres de forma violenta para poder consensuar sus estructuras de poder. No se puede decir que la obra de Shakespeare sea feminista, dado que no hay una crítica explícita, pero sí podría ser caracterizada de subversiva, pues el simple hecho de mostrar una realidad cruel, pero cargada de ironía, hace que el espectador reflexione sobre ese monólogo final, y así, tras un análisis detenido del mismo se debe enfrentar a la realidad que le rodea, y por qué no, transformarla.

\section{La representación de The Taming of the Shrew (1947-2008)}

$\mathrm{Si}$ atendemos a las representaciones de la obra The Taming of the Shrew, podemos apreciar que dependiendo del montaje y la versión a la que asistamos, podremos interpretarla de una u otra manera. Al fijar nuestra atención en diferentes puestas en escena de esta obra, a lo largo de los siglos XX y XXI, se puede observar las diferentes interpretaciones que a través de la representación puede tener el texto. De la función llevada a cabo en Sevilla en 1947 la crítica escribió: «La comedia girando en derredor de la supremacía del marido en el hogar, es distraída y de corte bufo, por lo exagerado de las situaciones [...] Pero tiene un fondo aleccionador, exponiendo la consecuencia de que unos días de contrariedad, pero inflexible conducta, pueden llevar a la felicidad para toda una vida matrimonial» (Pathe, 1947). Por estar enmarcada en los primeros años del franquismo, en los que se vuelve a los valores cristianos tradicionales, es decir, años en los que la mujer pierde los derechos ganados durante la República y se produce una vuelta a la idea de la inferioridad natural, al silencio como forma de expresión y a la sumisión al hombre como forma de vida, es lógico que nos encontremos con esta interpretación de la obra de Shakespeare. En la representación llevada a cabo en 1971 por el grupo Young Vic vemos que la crítica resalta ya no el contenido de la obra shakesperiana, si no el montaje técnico y circense en el que primó la introducción de elementos de pantomima, cómicos... que tuvieron un efecto de distanciamiento brechtiano, alejando al público de la realidad que la obra muestra y acercando lo máximo posible el texto a la comedia (Corbalán, 1971). En 1990 nos encontramos con una representación en Grecia, pero realizada por un director catalán, Salvat, en la que la clave para representar la ironía final se produce cuando al llegar el momento del tan temido monólogo,

Katerina se planta de forma impertinente. Y nuevamente surge el teatro dentro del teatro, personajes rebelados contra sus autores y en este caso contra el director, recordando la obra de Pirandello. Empieza un diálogo entre la fierecilla y el propio Salvat, aparentemente furiosos. Ella le dice que ya está bien, que estamos en 1990 y que está cansada de decir tonterías [...] tras la pataleta del director, la actriz vuelve a su monólogo que representa con un fondo de ironía impertinente (Castiella, 1990).

Esta representación, a diferencia de las anteriores, hace una crítica explícita al tratamiento de la mujer en la obra, marcando, aún más si cabe, la plausible ironía del monólogo final. A partir de este momento parece que la crítica antimisógina va a estar más ligada a la representación de la obra 
shakesperiana, ayudándose el director de diferentes elementos que la refuercen. Ejemplo de ello es el montaje de 1993 por la compañía La Trapera en Navarra, en el que se concedió el papel de Petruccio a una mujer; así «logra convertir una obra desfasada y machista en un elogio a la mujer de carácter y una crítica a la ñoñería que era el prototipo de mujer para los hombres del siglo XVII y de algunos individuos de nuestros días» (Oria, 1993). En 1999 nos encontramos con el montaje representado en el Corral de Comedias de Almagro, en el que «un gesto, la mano de Catalina dirigida con fuerza a las partes nobles de Petrucio es el que marca la tesis modernista-feminista de Carlos Marchena» (Herrero, 1999). Como se puede comprobar, la evolución de la representación de la obra es clara. Cada vez se intenta afianzar que el sentido final de la obra no debe ser misógino, recalcando así, en nuestra opinión, ese efecto que quiere conseguir la obra de exposición del público a una realidad que a veces parece oculta. Esta idea es exactamente con la que juega el director Edward Hall y su grupo teatral Watermill Theatre en su puesta en escena de 2006: «Los miembros de la compañía cuentan que la obra ha tenido una recepción difícil en Reino Unido, porque se la considera políticamente incorrecta y se trata mal a las mujeres [...] "Si les resulta difícil mirar, mejor", señala el intérprete [Scarfield]» (Bagué, 2006). Se expone al espectador a la violencia doméstica de forma brutal, y consideramos que al representar la violencia en el escenario se conmueve al público y se le enfrenta directamente con un problema que existía tanto en la época de Shakespeare como en la nuestra. Marta Monedero, en el diario Avui, califica esta representación incluso de feminista:

[...] l'adaptació d'una de les primeres comèdies del bard, L'amansiment de la fúria, en què els actors, tots homes, interpreten també els personatges femenins $[\ldots]$ «l'obra és difícil de representar perquè aborda la violència domèstica» [...] «Diria que a Shakespeare el coneixem una mica, ho aboca tot en el text i no se'l pot entendre sense conèixer la seva ironia». Posats a dir, l'actor no es talla: «És una obra feminista», tot $\mathrm{i}$ admetre'n «la crueltat». A l'escenari, «la violència s'aguditza de tal manera que és gairebé real, si la intèrpret fos una actriu, no es podria fer» (Monedero, 2006).

Finalmente no queremos dejar de mencionar la función de 2008 en el Corral de Zapateros, Alcalá de Henares, de Mariano de Paco Serrano, en el que directamente se elimina el monólogo final y que parece centrarse más en la oposición realidad/sueño:

Un montaje que termina corrigiendo lo concebido por Shakespeare, quien concluye la comedia cuando concluye la representación [...] De Paco hace que el infeliz Sly regrese a escena para darse de bruces con esa otra realidad de deudas, taberneros y un hogar poco dulce, no vaya nadie a creer que la vida es sueño. Se elimina así el monólogo de Catalina aconsejando a las mujeres que respeten a sus maridos, trufado de sutil mala leche (García Garzón, 2008)

Para concluir, queremos mencionar una de las representaciones más impactantes de esta obra, montada en Turquía y realizada bajo la dirección de Yücel Erten, en la que tras el monólogo final, Kate deja caer el chal que le cubre para mostrar que se ha cortado las venas (Shakespeare, 2010: 123).

\section{Conclusiones}

Como conclusión al análisis realizado sobre The Taming of the Shrew, se podría decir que la obra presenta una complejidad prácticamente inapreciable en una primera lectura y desde una perspectiva 


\section{Alicia Romero López}

actual. La misoginia que expresa Kate en el monólogo final no debe ser tomada al pie de la letra y siguiendo las claves expuestas anteriormente, el texto puede ser analizado con mucha más profundidad. Podemos decir que es una obra en la que se representa la «guerra entre sexos» pero, en nuestra opinión, consideramos que esto tan solo es la punta del iceberg. Shakespeare no solo nos muestra la relación habitual de la época entre hombres y mujeres (matrimonios concertados, violencia contra las mujeres, desprecio de todas aquellas que se impusieran a las normas de conducta exigidas...), sino que enfrenta al espectador a esa realidad. Al exponernos a una realidad a través de la comedia, de la ironía y del juego entre sueño y realidad (se ha de recalcar la importancia de la Induction) hace que el espectador vea con comicidad ese monólogo final de Kate, que sea capaz de reírse de esos valores misóginos presentes en la sociedad, quizá de forma inconsciente, pero logra que la sociedad a través de la ironía subvierta los valores establecidos. Sin embargo, no consideramos que ésta sea una obra feminista per $s e$, ya que la representación de la misma determinará el cariz que se le quiera dar. En este breve texto hemos ejemplificado algunos de los montajes más conocidos y se ha mostrado cómo hay muchas formas de representar la obra, lo que hace que cada una de ellas pueda tener una interpretación diferente. Además, al estar nosotros insertos en una sociedad patriarcal el monólogo puede ser entendido literalmente, como vimos en la representación de 1947. Es por ello que muchos de los directores introducen elementos propios para marcar bien esa ironía y comicidad shakesperiana, para no dejar lugar a la duda y para que finalmente sí podamos comprender la complejidad del texto y comprender que los valores de sumisión y subyugación de la mujer al hombre son valores que hace mucho tiempo debieron quedar obsoletos.

\section{Referencias bibliográficas}

AguirRe, M. E. (2012): «La fierecilla domada: la simulación como estrategia de supervivencia», en Actas del II Congreso Interdisciplinario sobre Género y Sociedad: "Lo personal es político". Córdoba, Universidad Nacional de Córdoba.

BAGUÉ, G. (2006): «Propeller lleva a Girona una "fierecilla domada" con violencia doméstica», El País (16-11-2006).

BALDwin, T. W. (1944): William Shakespeare's Small Latine and Lesse Greeke, en http://catalog. hathitrust.org/api/volumes/oclc/359037.html (última consulta, 20-4-15)

BIBLIA DE JERUSALÉN (1976): Biblia de Jerusalén. Ed. Jose Ángel Ubieta. Bilbao, GRAFO.

BLOOM, H. (1998): Shakespeare: The Invention of the Human, en https://www.nytimes.com/books/ first/b/bloom-shakespeare.html (última consulta, 29-4-15)

Booth, W. C. A. (1974): Rhetoric of Irony. Chicago, The University of Chicago Press.

Callaghan, D., ed. (2001): A Feminist Companion to Shakespeare. Malden, Blackwell.

CASTIElla, B. (1990): «Grecia. La fierecilla domada. Entente mediterráneo», El Público, 80, pp. 119120. 
Chalmers, A., ed. (1814): The General Biographical Dictionary. London, Nichols, Son \& Bentley, vol. XVIII.

CORBALÁN, P. (1971): «La fierecilla domada interpretada por el grupo Young Vic», Informaciones (12-10-1971).

Costa, M. (2012): «The Taming of the Shrew: "This is not a woman being crushed"», The Guardian (17-1-2012), en http://www.theguardian.com/stage/2012/jan/17/taming-of-the-shrew-rsc (última consulta, 30-4-15).

Davis, N. Z. (2006): «Mujeres y Política.», en G. Duby y M. Perrot, eds., Historia de las mujeres. Madrid, Taurus, vol. III.

Detmer, E. (1997): «Civilizing Subordination: Domestic Violence and The Taming of the Shrew.», Shakespeare Quarterly, 48/3, pp. 273-294.

ETON College (s. f.): «A brief history of Eton College», en http://www.etoncollege.com/briefhistory.aspx (última consulta, 24-4-15).

GARCÍA GARZÓN, I. (2008): «La fiera es sueño», $A B C$ (3-10-2008).

GASSnER, J., y QuinN, E., eds. (2002): The Reader's Encyclopedia of World Drama. New York, Courier Dover.

HIERON, S. (1620): The Sermons of Master Samuel Hieron: Formerly Collected Together by Himselfe, and Published in One Volume in His Life Time ... London, John Beale.

Herrero, F. (1999): «Modernidad», El Norte de Castilla (12-8-2009).

Don JuAn MANuel (2003): El Conde Lucanor o Libro de los enxiemplos del Conde Lucanor et de Patronio. Madrid, Castalia.

KeEble, N. H, ed. (2002): Cultural Identity of Seventeenth Century Woman. London, Routledge.

KEMP, T. D. (2010): Women in the Age of Shakespeare. California, ABC-CLIO.

Mikesell, M. L. (1990): «Love Wrought These Miracles: Marriage and Genre in The Taming of the Shrew», en M. B. Rose, ed., Essays on Dramatic Traditions: Challenges and Transmissions, Cambridge, Northwestern University Press.

Monedero, M. (2006): «Fúria de Shakespeare», Avui (16-11-2006).

ORIA, J. (1993): «Rescatar y ensalzar valores.», Navarra Hoy (20-4-1993).

PATHE. (1947): «Función en homenaje a Fernando Granada.», en ABC Sevilla (26-4-1947).

RocA, I. , y Gómez-HortigüelA, Á, eds. (2001): Juan Luis ViVes, Introducción a la sabiduría. Valencia, Ajuntament de Valencia.

SHAKESPEARE, W. (1994): Comedias. Barcelona, RBA.

_ (2008): The Taming of the Shrew. Ed. D. Callaghan. New York-London, W. W. Norton. (2010): The Taming of the Shrew. Ed. B. Hodgdon. London, Arden Shakespeare.

SMith, E. (2008): Shakespeare's Comedies: A Guide to Criticism. Cornwall, Blackwell.

RocA, I. Y A. GómeZ-HortigüElA. (2001): Introducción a la sabiduría de Juan Luis Vives, en http://bivaldi.gva.es/corpus/unidad.cmd?idCorpus=1\&idUnidad=11620\&posicion=1 (última consulta, 30-4-15). 
334 Tropelías. Revista de Teoría de la Literatura y Literatura Comparada, 25 (2016)

Alicia Romero López

RACKIN, P. 2005. Shakespeare and Women. New York, Oxford University Press.

ViveS, J. L. (1994a): La formación de la mujer cristiana. Trad. y ed. J. Beltrán Serra, en http://bivaldi.gva.es/es/corpus/unidad.cmd?idCorpus=1\&idUnidad=10066 (última consulta, 30-4-15).

_ (1994b): Los deberes del marido. Trad. y ed. de C. Bernal, en http://bivaldi.gva.es/en/corpus/ unidad.cmd?idUnidad=10109\&idCorpus=1 (última consulta, 30-4-15).

WATson, F. (1912): Vives and the Renaissance Education of Women, en http://hdl.handle.net/10111/ UIUCBB: watfor0001vivand (última consulta, 30-4-15).

WAYne, V., ed. (1991): The Matter of Difference: Materialist Feminist Criticism of Shakespeare. New York, Harvester Wheatsheaf. 